Cahiers d'études italiennes

21 | 2015

FILIgRANA

L'Italie et l'Orient : échanges, enjeux, regards croisés

\title{
E. S. Piccolomini et les Turcs : l'Europe face à son ennemi
}

E. S. Piccolomini e i Turchi: l'Europa di fronte al suo nemico

E. S. Piccolomini and the Turks: Europe against His Enemy

\section{Serge Stolf}

\section{OpenEdition}

\section{Journals}

Édition électronique

URL : http://journals.openedition.org/cei/2696

DOI : $10.4000 /$ cei.2696

ISSN : 2260-779X

\section{Éditeur}

UGA Éditions/Université Grenoble Alpes

Édition imprimée

Date de publication : 5 novembre 2015

Pagination : 105-117

ISBN : 978-2-84310-308-7

ISSN : $1770-9571$

\section{Référence électronique}

Serge Stolf, «E. S. Piccolomini et les Turcs : l'Europe face à son ennemi », Cahiers d'études italiennes

[En ligne], 21 | 2015, mis en ligne le 01 janvier 2017, consulté le 27 mars 2021. URL : http:// journals.openedition.org/cei/2696 ; DOI : https://doi.org/10.4000/cei.2696 


\title{
E.S. PICCOLOMINI ET LES TURCS : L'EUROPE FACE À SON ENNEMI
}

\author{
Serge Stolf \\ Université Grenoble Alpes
}

Le titre choisi pour cette brève étude, dans son raccourci, met en jeu une situation de conflit - celle de l'avancée militaire ottomane en terre d'Occident au Xve siècle - et un interprète de ce conflit, Enea Silvio Piccolomini, Siennois d'origine, humaniste aux intérêts éclectiques, poète, historien de l'Europe et, partiellement, de l'Asie, passé à l'état ecclésiastique, cardinal, puis pape (sous le nom de Pie II) de 1458 à I464. Il inscrit également les jugements de Piccolomini sur les Turcs dans des perspectives polémiques (comment pactiser avec une idéologie conquérante?), géopolitiques et géoculturelles. Il faut également ajouter le rôle éminent dont s'investit l'Église - le Siège romain — dans ce conflit. La réalité vécue par Piccolomini-Pie II, et par nombre de ses contemporains — avec plus ou moins d'acuité intellectuelle ou politique, selon les intérêts en jeu - est celle d'une guerre, et d'une confrontation avec l'Autre, identifié d'abord comme une altérité menaçante et, comme nous le verrons, infrahumaine, cruelle et barbare. Plusieurs études en ont donné une analyse riche et documentée ${ }^{1}$. Mais notre objet se limitera à une question qui ne cesse de revenir dans les pages de Piccolomini : qu'est-ce qui est menacé?

I. Signalons, sans prétention exhaustive : N. Housley (éd.), Crusading in the Fifteenth Century. Message and Impact, Houndmills-Basingstoke (Hampshire), Palgrave Macmillan, 2004; N. Bisaha, Creating East and West: Renaissance Humanists and the Ottoman Turks, Philadelphie, University of Pennsylvania Press, 2004; F. Cardini, "Sacro e menzogna. Crociata e propaganda antimusulmana nell'età di Pio II", dans L. Secchi Tarugi (éd.), Il sacro nel Rinascimento, Florence, Franco Cesati editore, 2000, p. 63I-659; D. Banks et M. Frassetto (éd.), Western Views of Islam in Medieval and Early Modern Europe: Perception of Other, New York, St. Martin's Press, 1999; L. F. Smith, "Pope Pius's Use of Turkish Atrocities», The Southwestern Social Science Quarterly, vol. 46, 1966, p. 407-415; C. A. Patrides, "The bloody and Cruell Turke”: The Background of a Renaissance Commonplace», Studies in the Renaissance, vol. X, 1963, p. I26-135 (l'article analyse ces préjugés dans des textes anglais des $\mathrm{XVI}^{\mathrm{e}}$ et $\mathrm{XVII}^{\mathrm{e}}$ siècles). 
Que s'agit-il de défendre face à un ennemi déterminé à détruire les fondements de l'Europe? Dans la réponse à cette question, Enea Silvio va définir une identité culturelle où l'héritage latin (et grec), l'Italie et les valeurs de l'humanisme profane et chrétien — et la centralité du Siège romain - tiennent une place déterminante.

Dans la prise de conscience des enjeux de ce conflit pour le monde européen et chrétien, il y a un avant et un après la chute de Constantinople, la «seconde Rome». L'intérêt de la très riche correspondance de Piccolomini, pendant les années passées au service de l'empereur Frédéric III de Habsboug ${ }^{2}$, est de faire percevoir les étapes de cette conscience chez celui qui deviendra un des plus fervents propagandistes d'une résistance à l'expansionnisme turc en Europe. Mais c'est au temps où Piccolomini se trouve encore au concile de Bâle et où les Grecs, sous la pression de plus en plus insoutenable des Ottomans, viennent plaider l'union avec Romes, qu'il prononce un discours où il souligne les risques de déstabilisation de la Chrétienté que fait courir l'avancée turque en Europe :

Grande est l'hégémonie des Turcs, considérables les forces asiatiques et éclatante leur puissance, ils ont étendu leur domination d'Asie en Europe, ils ont occupé toute la Grèce, comme s'ils habitaient les ruines de Troie, et, pour les chasser de la Grèce, il n’y faudrait pas un seul État ou une seule puissance, mais la Chrétienté tout entière4.

En considérant avec Piccolomini que le Bosphore est le «seul espace séparant l'Europe de l'Asie`", l'occupation d'une partie du continent européen, dès la fin du XIV siècle, transgresse cette frontière physique. Les armées du sultan ${ }^{6}$ ont atteint le Danube, et, après la défaite des Serbes à

2. E.S. Piccolomini entre au service de l'empereur d'Allemagne Frédéric de Habsboug (il n'est pas encore couronné) en I442, comme secrétaire à la chancellerie. Il deviendra par la suite un conseiller apprécié, mais pas toujours écouté, de son maître, et conservera des liens avec celui-ci pendant son cardinalat et jusqu'à son avènement au pontificat en 1458 .

3. Cette négociation, menée entre les délégués grecs et le pape Eugène IV, à Ferrare d'abord puis à Florence, aboutira à un décret d'union (6 juillet I439) des Églises grecques et latines. Le décret sera désavoué dès le retour de la délégation à Constantinople. Outre une aide financière pour la défense de la capitale byzantine, Eugène IV suscitera une campagne militaire qui aboutira au désastre de Varna, face aux Turcs. Voir F. Cardini, "Le concile de Ferrare et la croisade", Cahiers de recherches médiévales et humanistes, n ${ }^{\circ}$ I, 1996, p. 45-52. Sur le concile d'union de Florence, quelques vues d'ensemble dans J. Chélini, Histoire religieuse de l'Occident médiéval, Paris, Hachette, «Pluriel», I991, p. 552-557.

4. Pii II P. M. olim Enea Syluii Piccolominei Orationes politica et ecclesiastica, éd. J. D. Mansi, Lucæ, I755I759, pars I, p. II : "Magnum est imperium Turcorum, ingentes Asiaticorum vires, et opes ipsa florentissima, qui ex Asia in Europa imperium prorogarunt, totamque Greciam occuparunt tanquam Trojance cultores ruine, quos Gracia pellere non unius civitatis aut dominii, sed totius esset Christianitatis opus. " (Oratio habita Basilea pro loco eligendo ad recumenicum concilium cum Grecis celebrandum, p. 5-34)

5. Enee Silvii Piccolominei De Europa, ed. Adrianus van Heck, Città del Vaticano, Biblioteca Apostolica Vaticana, 200I, p. 7I (\$ 29) : "tantum [est] spacium, quod Europam Asiamque disterminat».

6. Se succèdent : Mourad I ${ }^{\text {er }}$ (I360-I389), Bayezid Ir (I389-I402), Mehmet I ${ }^{\text {er }}$ (I4I3-I42I), Mourad II (I42II45I), Mehmed II (I45I-I48I). 
la Maritza (septembre I37I), occupé la plus grande partie de la Bulgarie et la Macédoine serbe, avec importation de population. L'invasion des

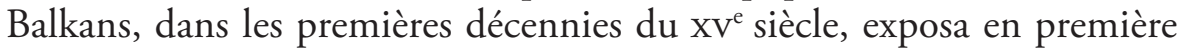
ligne la Hongrie, la Serbie, la Bosnie et les possessions vénitiennes en Égée. Le souvenir de la défaite des princes "croisés» à Nicopolis ${ }^{7}$ (septembre 1396) est présent dans les lettres où Piccolomini rapporte la nouvelle de la bataille de Varna, sur les bords de la mer Noire (novembre I444) - ultime tentative pour s'opposer par une action commune et offensive à l'avancée turque : 40000 Hongrois et Polonais, sous la conduite de Wladislaw Jagellon, roi de Hongrie, et de Jean Hunyade, sont anéantis par les armées du sultan Murad II ${ }^{8}$. Piccolomini a été hostile à ce parti de la lutte à outrance contre les Turcs, pour des raisons de réalisme politique : la mort de Jagellon permettra aux Habsbourg de voir renforcée leur position en Hongrie, nation sur laquelle ils avaient des prétentions hégémoniques. Les intérêts politiques immédiats prennent le pas sur ceux de la "croisade»".

Quant au sort de Constantinople et de l'Empire byzantin agonisant, il ne suscite dans ses lettres, à ce moment-là, nulle expression d'une urgence à mobiliser des forces militaires pour leur défense, comme si l'Occident n'avait pu croire cette mort inéluctable. Aucune imputation d'une responsabilité des Grecs dans leurs malheurs ne trouve d'écho dans les écrits d'Enea, même après la chute de Constantinople où certains verront une punition divine infligés aux Grecs «schismatiques»" ${ }^{10}$. Des puissances occidentales comme Venise ou Gênes se souciaient peu, au nom de leurs intérêts commerciaux, d'apporter leur soutien à une offensive d'envergure contre les Turcs.

Une littérature "antiturcica» commence à se diffuser après Varna, développant des lieux communs : les Turcs étaient la punition divine infligée aux chrétiens pour leurs péchés, ils étaient cruels, mais la Hongrie était «le rempart et le bouclier» (murus et clipeus) de la Chrétienté ${ }^{I I}$, comme

7. Sur la rive sud du Danube (aujourd'hui en Bulgarie).

8. Varna, qualifiée par les historiens de l'Europe de l'Est de «bataille des siècles" rappelait la défaite à Nicopolis, le 23 septembre 1396, face aux Ottomans, d'une armée de plusieurs milliers de croisés.

9. Piccolomini considère d'ailleurs la défaite comme un effet de la justice divine, car certains chrétiens ne sont venus se battre que pour un butin, une solde ou la gloire. Voir R. Wolkan, «Der Briefwechsel des Aeneas Silvius Piccolomini», dans Fontes Rerum Austriacarum : Diplomataria et Acta, vol. 67, Vienne, I9I2 [comprenant les lettres d'E. S. Piccolomini de I447 à I450], p. 72-77 (lettre du 25 novembre I448 à Nicolas V).

Io. N. Agapiou, «Enea Silvio Piccolomini et les Grecs», dans Pio II umanista europeo (Atti del XVII Convegno Internazionale, Chianciano-Pienza, I8-2I juillet 2005), Florence, Franco Cesati editore, 2007, p. I45-I64.

II. Voir K. Pajorin, "I primordi della letteratura antiturca in Ungheria e Pio II", dans Pio II umanista, ouvr. cité, p. 815-827. 
le répète Piccolomini ${ }^{12}$. Cette rhétorique méconnaissait toutefois la réalité des rapports de force et sous-évaluait la pugnacité des Turcs. Une lettre de Filelfo au roi de France, Charles VII, présente le nouveau sultan Mehmet II comme un être amolli dont il serait aisé de venir à bout militairement ${ }^{13}$. L'amollissement de ces adversaires, produit supposé d'une lubricité naturelle, est un poncif que Piccolomini réutilisera dans sa lettre à Nicolas V, écrite peu de semaines après la prise de Constantinople : «Ô honte! nous avons permis à ces efféminés de Turcs de prendre la noble cité de Constantinople ${ }^{14}$.» Mais il est clair que la Chrétienté est une citadelle assiégée par l'"Antéchrist». Georges de Trébizonde, dans un discours d'octobre I452 adressé à Nicolas V, Pro defendenda Europa et Hellesponti claustra, veut démontrer que Constantinople est la dernière défense des détroits du Bosphore, c'est-à-dire de l'Europe ${ }^{\text {Is }}$. Cependant, dès I397, Coluccio Salutati faisait une autre lecture de cette confrontation : voyant dans le Grand Schisme l'une des causes de la vulnérabilité des chrétiens aux attaques des Turcs, gens endurants et disciplinés, il insistait sur la nécessité que la «civilisation" se ressaisît moralement face aux barbares ${ }^{16}$. Enea, lui aussi, mettra en cause la décadence morale et politique de l'Occident.

La nouvelle de la prise de Constantinople parvint à Venise le 29 juin I453. Le I2 juillet, de Graz, Enea adressait sur cet événement une longue lettre ${ }^{17}$ au pape, Nicolas V. La rapidité de la chute ne laisse pas de l'étonner, non moins que l'indifférence coupable des nations chrétiennes. Il donne le chiffre de "plus de quarante mille personnes" passées au fil de l'épée ${ }^{18}$. Il s'attarde sur les destructions des églises et des bibliothèques :

Je souffre à la pensée des nombreuses basiliques consacrées aux saints, constructions d'un admirable travail, soumises à la destruction ou à la souillure de Mahomet. Que dirais-je des livres innombrables qui se trouvaient en ce lieu, encore inconnus aux

I2. Enee Siluii Piccolominei Epistolarium seculare, complectens De duobus amantibus, De naturis equorum, De curialum miseriis, post R. Wolkan iterum recognouit edidit Adrianus van Heck, Città del Vaticano, Biblioteca Apostolica Vaticana, 2007 p. 473 : «murus eius [religio christiana], qui est Hungaria».

I3. F. Cardini, "Sacro e menzogna. Crociata e propaganda antimusulmana nell'età di Pio II », dans L. Secchi Tarugi (éd.), Il sacro nel Rinascimento, Florence, Franco Cesati editore, 200o, p. 643. La lettre est du 20 mars I45I.

I4. R. Wolkan, art. cité, vol. 68, p. 199 (lettre du I2 juillet I453 à Nicolas V) : «[... proh pudor ab effeminatis Turchis inclitam urbem Constantinopolim capi permittimus."

15. A. Pertusi, La caduta di Costantinopoli. Le testimonianze dei contemporanei. L'eco nel mondo, Milan, Fondazione L. Valla-A. Mondadori, 1976, vol. I, introd. p. XıII.

I6. M. Meserve, "Italian Humanists and the Problem of the Crusade», dans N. Husley, Crusading in the Fifteenth Century, ouvr. cité, p. I6-I7.

17. R. Wolkan, art. cité, vol. 68, p. 189-202.

I8. Ibid., p. 199 : "populum omnem gladio extinxit [...] quadraginta et amplius milia personarum illic occisa referuntur». S. Runciman (The Fall of Constantinople 1453, Paris, Tallandier, 2007) établit à 4000 le nombre des victimes (p. 217). 
Latins? Las, combien de noms de grands hommes périront à présent? C’est la seconde mort d'Homère, le second trépas de Platon. Où rechercherons-nous les œuvres de génie des philosophes et des poètes? La source des Muses est tarie, puisse-t-il nous rester assez de talent pour pouvoir pleurer ce grand malheur avec de dignes accents ${ }^{19}$.

Lévénement fait surgir la conscience — bien soudaine — d'une perte irréparable pour la civilisation de l'Europe, d'une amputation de son patrimoine culturel, la coupure de cette translatio des lettres de la Grèce à l'Europe (au moment où Nicolas V faisait traduire des manuscrits grecs), et l'image du glaive forçant «le domicile des lettres» laisse planer la crainte d'une dévastation plus totale :

En Europe, sur notre sol, nous avons laissé l'ennemi s'emparer de la plus puissante ville de la Chrétienté, la capitale de l'Empire d'Orient, la colonne de la Grèce, le domicile des lettres [litterarum domicilium]. Désormais Mahomet règne parmi nous. Sur nos têtes est désormais suspendu le glaive des $\operatorname{Turcs}^{20}$.

Cette lettre, très importante dans la mise en place par Piccolomini d'une rhétorique de la "croisade» contre les ennemis de la civilisation, contient un des thèmes constamment développé pendant son pontificat par Piccolomini : la sauvegarde de la foi va de pair avec celle du foyer de civilisation européenne, édifiée sur Rome et sur le Christ. Dans la lettre adressée, le 2I juillet suivant, à Nicolas de Cues, il va jusqu'à faire un parallèle entre les Perses, barbares de l'Antiquité, qui «firent la guerre aux hommes et non à la littérature ${ }^{2 \mathrm{I}} »$, et les Turcs, barbares modernes :

À présent, il en ira autrement sous la domination des Turcs, hommes d'une extrême cruauté, ennemis des bonnes mœurs et de la culture [...]. Ils se roulent dans la débauche, tiennent en piètre estime l'étude des lettres, sont incroyablement orgueilleux ${ }^{22}$.

Ce thème de l'obscurantisme des Turcs est amplement développé dans la littérature humaniste de l'époque, et N. Bisaha souligne la réversibilité de cette accusation, en citant l'historien ottoman Sa'd ed Din qui écrivit

19. R. Wolkan, art. cité, vol. 68, p. 200 : "doleo infinitas sanctorum basilicas opere mirando constructas vel ruine vel spurcitie Mahometi subjacere. quid de libris dicam, qui illic erant innumerabiles, nondum Latinis cogniti? heu, quot nunc magnorum nomina virorum peribunt? secunda mors ista Homero est, secundus Platoni obitus. ubi nunc philosophorum aut poetarum ingenia requiremus? extinctus est fons musarum, utinam tantum nobis superaret ingenii, ut hanc calamitatem dignis vocibus deplorare possemus. "Si toutes les églises ne furent pas détruites, la plupart des livres furent brûlés (S. Runciman, ouvr. cité, p. 216-217).

20. R. Wolkan, art. cité, vol. 68, p. 201 : "nos in Europa in nostro solo, inter Christianos potentissimam urbem, orientalis imperii caput, Grecie columen, litterarum domicilium ab hostili manu sinimus expugnari. iam regnat inter nos Mahometus. iam nostris cervicibus imminet Turcorum gladius. "

2I. Ibid., p. 209 : «bellum viris, non litteris intulerunt».

22. Ibid., p. 209-210 : "nunc sub Turchorum imperio secus eveniet, sevissimorum hominum, bonorum morum atque litterarum hostium [...]. in libidinem provoluti sunt, litterarum studia parvi faciunt, incredibili fastu superbiunt." 
au XVI ${ }^{\mathrm{e}}$ siècle. Celui-ci rappelle que la conquête de Constantinople, en transformant les temples des "mécréants" ("misbelievers») en mosquées, "a chassé les hôtes des ténèbres" ("drove away the hosts of darkness") ${ }^{23}$. Quelques années plus tard, en I458, dans son De Europa, Piccolomini s'attachera à définir ce qui sépare les Européens des barbares. Au terme d'un rapide survol des conquêtes turques au début $\mathrm{du} \mathrm{Xv}^{\mathrm{e}}$ siècle, marquées par les pillages et les incendies, il voit dans leur haine de la culture hellénique la meilleure preuve de leur barbarie. Il réfute l'opinion de ceux qui, en raison de l'occupation de l'Asie mineure par les Ottomans, attribuent à ceux-ci une ascendance troyenne dont, souligne-t-il, ils ne peuvent être en aucune manière les héritiers : «Les Troyens étaient originaires de Crète et d'Italie; la race des Turcs est scythe et barbare ${ }^{24}$.»

Ils ne peuvent donc se réclamer en aucune façon d'une filiation avec la civilisation antique, mais sont désignés, de ce fait, comme les ennemis de l'humanité, de l'humanitas, définie par la raison et l'amour des lettres.

La prise de Constantinople signifie que la puissance turque est devenue un problème éminemment européen, un danger pour le rayonnement de sa foi et de sa civilisation. Relayant l'idée exprimée par Coluccio Salutati sur le danger que représente la division de la Chrétienté face au péril turc, et que nous avons citée plus haut, Piccolomini se saisit de la situation italienne pour dénoncer ce danger. Le jeu des alliances scellées dans le seul but des intérêts particuliers de tel État au détriment de tel autre, doit laisser place au seul intérêt général, celui de la croisade défensive contre l'ennemi commun à l'Europe chrétienne. Des symptômes se font jour, tel le relâchement de la foi, annonciateurs d'une ruine morale qui précipitera la fin de sa civilisation. Dans sa lettre à Leonardo Benvoglienti, ambassadeur de la République siennoise à Venise, datée du 25 septembre I453, Enea appelle au ressaisissement des vertus sans lesquelles la colère de Dieu, dont les Turcs sont l'instrument, s'abattra sur les chrétiens :

Nous n'honorons pas les parents et nous n'enseignons pas de choses honnêtes à nos enfants. Nous nous rebellons contre les maîtres, nous sommes hautains envers les serviteurs. Nous avons en vue la cupidité, nous courons après la débauche, nous sommes esclaves du plaisir. Nous ne rendons aucun culte à l'innocence. Quoi d'étonnant si

23. N. Bisaha, Creating East and West..., ouvr. cité, p. 74 (Chapter 2. The New barbarian). L'auteur, à la suite de cette citation, commente: "While the theme here is clearly religious, the symbolism of the language is eerily similar to that of humanists describing the Turks' culture as one of darkness, versus the splendor of (Western) freedom and enlightenment [lumières]."

24. E. S. Piccolomini, De Europa, ed. Adrianus van Heck, Città del Vaticano, Biblioteca Apostolica Vaticana, 200I, p. 62-63 (\$ 20-2I) : "sed illorum origo ex Chreta atque Italia fuit; Turcorum gens scythica et barbara est." Les Scythes, originaires d'Iran, peuplaient dans l'Antiquité les steppes au nord de la mer Noire. 
la colère de Dieu contre nous augmente la puissance turque et suspend au-dessus de nos têtes le glaive ennemi ${ }^{25}$ ?

À partir du De Europa (I458), Piccolomini va consolider sa rhétorique sur la «barbarie» de l'ennemi commun. Nous en avons déjà noté plusieurs traits. Cette rhétorique ne lui appartient pas en propre, il en hérite de cette littérature humaniste dont il partage les points de vue quant à la distinction séparant irrémédiablement l'espace de la civilisation — l'Europe de celui de la barbarie. Il a pu puiser également dans un texte qu'un humaniste, né en Eubée, connu sous le nom latin de Niccolò Sagundino (première décennie $\mathrm{du} \mathrm{Xv}$ siècle-I464), lui avait adressé lorsqu'il était évêque de Sienne, en I456. Ce texte, intitulé Epistula Nicolai Sagundini de familia Otumanorum, retrace une brève histoire des origines scythes du peuple turc et de ses conquêtes ${ }^{26}$. Il faut par ailleurs accepter l'idée qu'une "propagande» fût alors nécessaire pour inciter des nations chrétiennes qui, en dehors de celles qui se trouvaient sur la ligne de front de la pression militaire ottomane, se sentaient moins concernées. Il importait de définir dans l'ennemi les caractéristiques d'une barbarie prête à submerger l'Europe et sa culture, puisque, déjà, Athènes n'est plus (prise de l'Acropole, juin 1458), la Morée (le Péloponnèse) est dévastée par les armées de Mehmet II au cours de l'année I458, la Serbie sur le point de succomber ${ }^{27}$.

L'événement de Constantinople, le bastion que l'on croyait imprenable, continue de résonner dans la conscience de Piccolomini. Il est une évidence que le De Europa, description géo-historique de l'Europe, commence par la péninsule balkanique, là où se concentraient les problèmes majeurs du moment. Ainsi, la région qu'il appelle Romania (Roumanie) — en fait la Roumélie ${ }^{28}$ — occupée par les Turcs est un exemple d'une parcelle d'Europe retournée à son état originel d'avant la civilisation :

25. R. Wolkan, art. cité, vol. 68, p. 281 : "nam quid est, quod nos de lege divina servemus? que civitas non admittit usuras? quis jejunat, quis festos dies religiose colit? quot falsa testimonia, quot perjuria, quot adulteria dietim admittuntur? [...] nec parentes honoramus nec filios honesta docemus. in dominos rebelles, in servos superbi sumus. avaritie omnes studemus, libidinum sequimur, voluptati servimus. innocentie nullus est cultus. quid mirum, si furor domini supra nos excitatus Turchum elevat et inimici gladium super cervices nostras extendit?" (Lettre écrite à trois mois de la chute de Constantinople.)

26. Le texte est édité par A. van Heck dans E. S. Piccolomini, Carmina, Città del Vaticano, Biblioteca Apostolica Vaticana, I994, p. 217-225.

27. Sur l'avancée turque dans les Balkans, voir : M. Spremič, "I Balcani e la crociata (I455-I464)", dans Il sogno di Pio II e il viaggio da Roma a Mantova, Florence, Olschki, 2003, p. 48I-502.

28. Il s'agit de toute la zone maritime jusqu'à l'Hellespont (détroit des Dardanelles) correspondant à la Thrace et à la Macédoine. 
[Elle est] de nation grecque, bien qu'autrefois elle ait été barbare, et que, de nos jours, après la destruction de l'empire grec, sous la domination des Turcs, elle soit retournée à la barbarie ${ }^{29}$.

Il est tout aussi symptomatique que le De Europa, dont la deuxième partie décrit les États italiens, s'achève sur la royaume de Naples et sur l'appel à Alphonse d'Aragon, roi d'Aragon et de Naples, sur lequel la papauté, et Piccolomini lui-même, fondait ses espoirs pour prendre la tête d'une expédition militaire contre les Ottomans. En avril I457, il avait écrit au roi pour l'inviter à prendre la tête de la croisade : «Il n'est pas de roi, en effet, dans le monde chrétien qui ait plus d'autorité que toi ${ }^{30}$.»

Enea s'attache donc à définir clairement ce qui sépare les Européens des barbares. Il retrace l'histoire des conquêtes turques. Enea voit dans ces pillards et ces incendiaires, dans leurs mœurs et leur haine de la culture hellénique, la meilleure preuve de leur barbarie. À ceux qui leur attribuent une ascendance troyenne parce qu'ils occupent l'Asie Mineure, Enea répond qu'ils ne peuvent être les héritiers d'une race et d'une civilisation originaire d'Europe :

Les Troyens étaient originaires de Crète et d'Italie; la race des Turcs est scythe et barbare [...]. Les Turcs [sont] un peuple cruel, plein de turpitude, fornicateur, se donnant à tous les adultères et à toutes les débauches. Ils mangent des choses qui font horreur aux autres peuples : viandes de jument, de loup et de vautour ${ }^{31}$.

Cruauté naturelle, absence de morale, luxure, nourriture immonde, autant de traits qui les rejettent dans une sorte d'infra-humanité ${ }^{32}$. Piccolomini reprendra, dans Asia, écrit en juillet-août I463, des arguments qu'il avait déjà utilisés dans son discours à la diète de Ratisbonne, en avril I454 :

Et bien que pendant plusieurs siècles [ce peuple] se soit montré un peu civilisé sous l'effet de la douceur du ciel et de la fertilité du sol, cependant, aujourd'hui encore, il se ressent beaucoup de sa monstruosité originelle, et ne s'est pas purgé de toute barba-

29. E.S. Piccolomini, De Europa, ouvr. cité., p. 59 (\$ 18$)$ : "post quos [Bulgaros] loca maritima uersus meridiem ad Hellespontum usque Romania est, natio greca, quamuis olim barbara fuerit, et iterum nostro tempore deleto Grecorum imperio dominantibus Turcis in barbariam redit. "

30. Enea Silvio Piccolomini, Lettere scritte durante il cardinalato (I456-I457), Ettore Malnati (éd.), Brescia, Marco Serra Tarantola, 2007, lettre 8I : «Neque enim rex est in orbo Christiano apud quem plus auctoritatis quam apud te sit. " Peu après la chute de Constantinople, Flavio Biondo écrit deux textes où est affirmée la nécessité de la croisade : De expeditione in Turchos, dédié à Alphonse d'Aragon, et De origine et gestis venetorum (I454).

3I. De Europa, ouvr. cité, p. 62-63 (\$ 20-2I) : «sed illorum origo ex Chreta atque Italia fuit; Turcorum gens scythica et barbara est [...] [Turci] gens truculenta, ignominiosa et in cunctis stupris ac lupanaribus fornicaria. comedit que ceteri abominantur : iumentorum, luporum et uulturum carnes. "

32. Hérodote et Eschyle (Les Perses) identifiaient de même l'Asie comme le continent des barbares, par opposition à la Grèce civilisée : voir F. Cardini, Europe et islam. Histoire d'un malentendu, traduit de l'italien par J.-P. Bardos, Paris, Seuil, 2002 (édition italienne, 2000), p. 209-2IO. 
rie. Il se nourrit encore de viande de cheval, de putois et de vautour, est l'esclave de la débauche, succombe à la cruauté, déteste les lettres, persécute les études d'humanité33.

De Scythie sont originaires les hordes d'envahisseurs qui ont déferlé sur l'Occident : Huns, Goths, Lombards, Turcs enfin ${ }^{34}$, ultime avatar des Scythes. Déconsidérer l'adversaire sous les traits de la sauvagerie et de l'absence de civilisation permet de souligner la différenciation totale entre les Européens et leurs ennemis. À Mantoue où le pape Piccolomini-Pie II conviera les princes de l'Europe ou leurs représentants à participer à la croisade défensive contre les Turcs, l'humaniste Filelfo fera un discours qui reprend ces notations sur la sauvagerie native des Turcs :

Toute indignité, en effet, doit être regardée comme d'autant plus indigne qu'elle est infligée par des hommes plus indignes; si l'on peut appeler les Turcs des hommes plutôt que des bêtes particulièrement sauvages et cruelles, dans la mesure où il n'y a en eux aucune trace d'humain à part l'apparence extérieure ${ }^{35} \ldots$

Pour Piccolomini, le destin de l'Europe est scellé par le double héritage de la culture classique et du christianisme. L'agression externe permet d'affirmer l'unité de ses valeurs et de ses indispensables solidarités. Devenu pape Pie II, il se fera l'infatigable promoteur d'une action défensive contre les Turcs. À l'issue du congrès de Mantoue, il édicte la bulle Ecclesiam Christi (I4 janvier I460) proclamant la croisade contre les Turcs pour trois ans. L'accent est davantage mis sur la rhétorique de la défense de la foi chrétienne contre l'assaut de l'islam. Pie II écrira un poème intitulé "Contre Mahomet [II], sans foi, roi des Turcs», dont voici un extrait :

33. Aeneae SylVII PICCOLOmineI Senensis qui post adeptum Pontificatum Pius eius nomini Secundus appelatus est, opera quae extant omnia... / Basileae/ ex officina Henricpetrina, I57I, p. 68I (Oratio Aeneae de Constantinopolitam Clade, et bello contra Turcos congregando) : "Et quamuis sub miti coelo et mundiori terra per tot secula parumper excultam se praebuerit, sapit tamen adhuc multum pristinae deformitatis, neque omnem Barbariem detersit. Carnes adhuc equorum, uesontium, uulturumque comedit, libidini seruit, crudelitati succumbit, literas odit, humanitatis studia persequitur." Enea utilise, dans les deux textes, les informations tirées de la Cosmographia d'un géographe latin, Aethicus Ister ( $\mathrm{v}^{\mathrm{e}}-\mathrm{vI}^{\mathrm{e}}$ siècle). Sur l'exploitation qui est faite de la cruauté des Turcs lors de la prise de Constantinople, voir L. F. Smith, "Pope Pius II's use of Turkish Atrocities», The Southwestern Social Science Quarterly, vol. 46, I966, p. I04-II5.

34. Sur les Scythes, leur image dans l'Antiquité et l'exploitation qu'en fait Pie II dans l'Asia, voir M. Meserve, "From Samarkand to Scythia: Reinventions of Asia in Renaissance Geography and Political Thought», dans Pius II 'el più expeditivo pontefice', p. 13-39.

35. Cité par M. Meserve, "Italian Humanists and the Problem of the Crusade», dans Crusading in the Fifteenth Century, ouvr. cité, p. I86 : "Eo enim omnis indignitas ducenda est indignior, quo ab indignioribus infertur hominibus; si homines quidem potius quam efferatae prorsus et truculentissimae quaedam belluae Turci sunt appellandi, cum nibil in se humanitatis habeant praeter hominis figuram..." L'auteur explique que les humanistes, en opérant des citations sélectives à partir des sources médiévales et byzantines à leur disposition, ont produit "a portrait of the Turks as savage invaders, 'bursting through' the Caspian Gates in a suggestively catastrophic manner" (p. 29). 
Ô Turc, tu te prépares à renverser les hautes murailles de Rome/ et tu penses pouvoir détruire la loi du Christ. / Mais Pie marche contre toi; il exhorte les rois et les généraux à prendre rapidement les armes. Les voux de Pie sont sacrés [...]. Ils ne respectent ni la loi nouvelle [Nouveau Testament], ni l'ancienne,/ ils n'accueillent ni les paroles de Moïse, ni les tiennes, ô Jésus. / Ils croient que Mahomet est descendu du troisième ciel/ et que tu l'as inspiré. / Mais quelle honnêteté chez ce peuple, quelle morale dans sa $\operatorname{loi}^{36}$ ?

Ainsi la «Lettre à Mehmet», l'Epistola ad Mahumetem (début I462), que Pie II pensait peut-être adresser au sultan des Turcs, mais qu'il ne lui envoya jamais, ne cache nullement les intentions apologétiques de la religion chrétienne. L'Italie est prête à mourir pour sa foi, l'Église romaine n'est préoccupée que «de conserver et d'étendre la foi en Notre Seigneur Jésus ${ }^{37}$ ». Seul le baptême pourrait faire admettre le sultan comme empereur d'Occident. La lettre émane d'un esprit de dialogue, contre le courant d'opinion, diffus à partir du XIV ${ }^{\mathrm{e}}$ siècle, selon lequel disputer avec les musulmans, gens réfractaires à la raison, était inutile. Mais ce dialogue passe par une critique des fondements de l'islam, religion reposant sur le fidéisme et non sur la raison. Pour Pie II, la religion de Mahomet s'est imposée par la force des armes contre la raison, ce qui la dévalorise à ses yeux.

La réponse armée à l'agression turque demeure pour Pie II une solution à court terme, une réponse à l'agression, le coup de frein à l'avancée des Ottomans en Europe. Dans le passage qui suit, le recours à la métaphore de l'islam comme un édifice aux fondements fragiles qui tombera sous les «armes» de la Vérité et de la raison, constitue l'argument qui doit inciter les princes chrétiens à passer de la résistance à l'offensive, comme une réponse différée à l'écroulement de l'Empire byzantin.

Il [Mahomet] a opposé les armes à la raison, ordonnant que personne ne discute sa religion et ne cherche une explication rationnelle : ceux qui s'efforçaient de la contester, il fallait les châtier par le fer. Avec ce rempart, le vieux renard plein d'astuce fortifia sa loi. Mais ce n'est pas un mur de diamant, ni de fer, ni de pierre vive : c'est un matériau fragile. Cette maison protégée par l'argile et la boue sera facile à atta-

36. Carmina, p. I57-I58, v. I-4; v. 19-23: "Turche, paras alte subuertere menia Romel et Christi legem perdere posse putas. I it Pius at contra : reges capere arma ducesquel ocius hortatur. sunt pia uota Pii [...] nulla noue legis, nulla est reuerentia prisce, I nec Moysi recipit nec tua uerba, Iesu. I tercius ex alto Mahumetes missus olympo I creditur et flatu non caruisse tuo. I sed quenam probitas gentis? que legis honestas? (In Mahumetem perfidum Turchorum regem).» Ce poème, non daté, pourrait avoir été écrit à l'époque du congrès de Mantoue, quand se faisait sentir pour Pie II l'urgence de la croisade.

37. Epistola, dans L. D'Ascia, Il Corano e la tiara. L'Epistola a Maometto II di Enea Silvio Piccolomini (papa Pio II), Bologne, Pendragon, 200I, p. 236 : "[nobis] sola cura est servanda et amplianda fidei domini nostri Jesu». 
quer, et elle ne résistera pas à nos traits : les édifices construits sur le sable s'écroulent aisément ${ }^{38}$.

Dans la rhétorique déployée par Piccolomini — et pas seulement la rhétorique, puisqu'il s'efforcera de réunir jusqu'à son dernier souffle les forces matérielles et militaires nécessaires à la croisade - l'ennemi est clairement désigné comme l'agresseur. Il est défini par les traits les plus propres à souligner le danger bien réel d'asservissement qu'il représentait pour les peuples d'Europe. La mise en place d'une image, cultivant le mythe des Scythes nomades et donc barbares, soustrait au visage de cet ennemi les traits fondamentaux d'une appartenance à l'humanité. Elle le soustrait à la civilisation qu'il veut détruire, dont les limites sont celles d'une Europe héritière de la culture grecque, latine et chrétienne, où le savoir est mis publiquement à la portée de tous, où les arts libéraux sont partout cultivés. Cette réaction ressemble davantage à un sursaut, à une réaction de survie. Certains États envisageaient déjà des accommodements avec les Ottomans, tandis que d'autres résistaient. Piccolomini, dans les pages de ses Commentarii dictés pendant son pontificat, souligne le rôle éminent des souverains de Castille dans la Reconquista dont une des dernières étapes, de son vivant, est la prise de Gibraltar en I462 par les armées d'Henri IV de Castille. Cette «reconquête» [il emploie le mot latin recuperare] a une valeur symbolique :

Un fait est certain : c'est de nos jours seulement que Gibraltar est revenu de nouveau entre des mains chrétiennes, et que, remplaçant les temples de Mahomet, des églises sont à présent consacrées à la Piété divine et que de nombreux habitants chrétiens y ont afflué39.

La situation reste bien celle d'un affrontement. Nul ne sait comment la lettre à Mehmet II, si elle avait été réellement expédiée à celui-ci, eût été reçue. Le pont lancé vers l'Autre, l'ennemi, s'élance à mi-course, mais il est lancé.

38. Ibid., p. 27I : "Rationi arma obiecit, iubens ne quispiam de sua lege disputaret neve rationem quaereret: in eum, qui contra niteretur, ferro vindicandum esse. Atque huiusmodi vallo suam legem callidus veterator communivit. Sed non est hic adamantinus murus, neque ferreus, neque ex vivo lapide: fragili materia est. Creta et luto munita domus facile oppugnabitur, neque resistet telis nostris : facile ruunt edificia in arena fundata."

39. PII II, Commentarii rerum memorabilium que temporibus suis contigerunt, editi ab A. van Heck, 2 vol., Città del Vaticano, Biblioteca Apostolica Vaticana, 1984, vol. 2, X, 24, p. 616-617 : «certa res est nostro demum seculo ad christianas manus Gebeltarum rediisse et, ubi fuere Maumethis templa, nunc ecclesias diuine pietati dicatas esse et multos ex christianis habitatores eo confluxisse." 


\section{Bibliographie succincte}

Agapiou Natalia, "Enea Silvio Piccolomini et les Grecs», dans Pio II umanista europeo (Atti del XVII Convegno Internazionale, ChiancianoPienza, I8-2I juillet 2005), Florence, Franco Cesati editore, 2007, p. I45-I64.

Banks David et Frassetto Michael (éd.), Western Views of Islam in Medieval and Early Modern Europe: Perception of Other, New York, St. Martin's Press, I999.

Bisaha Nancy, Creating East and West: Renaissance Humanists and the Ottoman Turks, Philadelphie, University of Pennsylvania Press, 2004.

Cardini Franco, "Sacro e menzogna. Crociata e propaganda antimusulmana nell'età di Pio II", dans L. Secchi Tarugi (éd.), Il sacro nel Rinascimento, Florence, Franco Cesati editore, 2000, p. 63I-659.

D’Ascia Luca, Il Corano e la tiara. L'Epistola a Maometto II di Enea Silvio Piccolomini (papa Pio II), Bologne, Pendragon, 200 .

Housley Norman (éd.), Crusading in the Fifteenth Century. Message and Impact, Houndmills-Basingstoke (Hampshire), Palgrave Macmillan, 2004.

Meserve Margaret, «Italian Humanist and the Problem of the Crusade», dans N. Housley (éd.), Crusading in the Fifteenth Century. Message and Impact, Houndmills-Basingstoke (Hampshire), Palgrave Macmillan, 2004, p. I3-38.

PAJORIN Klara, "I primordi della letteratura antiturca in Ungheria e Pio II", dans Pio II umanista europeo (Atti del XVII Convegno Internazionale, Chianciano-Pienza, I8-2I juillet 2005), Florence, Franco Cesati editore, 2007, p. 815-827.

Patrides Constantinos A., "The Bloody and Cruell Turke": The Background of a Renaissance Commonplace», Studies in the Renaissance, vol. X, 1963, p. I26-I35.

Pertusi Agostino, La caduta di Costantinopoli. Le testimonianze dei contemporanei. L'eco nel mondo, Milan, Fondazione L. Valla-A. Mondadori, 2 vol., 1976.

PII II P. M. olim Anece Syluii Piccolominei Orationes politice et ecclesiastice, éd. J. D. Mansi, Lucæ, I755-I759.

Smith Leslie F., "Pope Pius's Use of Turkish Atrocities", The Southwestern Social Science Quarterly, vol. 46, 1966, p. 407-415.

Spremič Momčilo, "I Balcani e la crociata (I455-I464)», dans Il sogno di Pio II e il viaggio da Roma a Mantova, Florence, Olschki, 2003, p. 48I-502. 
STOLF Serge, «Espace géographique et espace culturel : le De Europa de Enea Silvio Piccolomini», Camenae, n ${ }^{\circ}$ I4, novembre 20I2, p. I-I7. Stolf Serge, Les Lettres et la Tiare. E. S. Piccolomini, un humaniste au $X V^{e}$ siècle, Paris, Classiques Garnier, "Études et essais sur la Renaissance», 2012. 